\title{
Interactive Reconstruction via Geometric Probing *
}

\author{
Steven S. Skiena ${ }^{\dagger}$ \\ Department of Computer Science \\ State University of New York \\ Stony Brook, NY 11794-4400
}

September 3, 1991

\begin{abstract}
Geometric probing considers problems of determining a geometric structure or some aspect of that structure from the results of a mathematical or physical measuring device, a probe. A variety of problems from robotics, medical instrumentation, mathematical optimization, integral and computational geometry, graph theory and other areas fit into this paradigm.

This paper surveys the field of geometric probing, with results ordered by probing model. The emphasis is on interactive reconstruction, where the results of all previous measurements are used to determine the orientation of the next probe so it provides the maximum amount of information about the structure. Through interactive reconstruction, finite determination strategies exist for such diverse models as finger, x-ray, and half-plane probes.
\end{abstract}

\section{Introduction}

Many problems from mathematics and engineering can be described in terms of reconstruction from projections. We define a projection to be a measurement of some aspect of a physical or mathematical object and reconstruction as the algorithmic problem of combining the result of several projections to obtain some desired information about the object.

Practical reconstruction problems include:

- Computer-aided Tomography - CAT scanners and other tomographic imaging systems represent a tremendous step forward in our ability to diagnose tumors and other medical problems. Herman [38] defines tomography as "the process of producing an image of a two-dimensional distribution (usually of some physical property) from estimates

${ }^{*}$ Invited paper, IEEE Proceedings.

${ }^{\dagger}$ This work was partially supported by NSF Award CCR-9109289. 
of its line integrals along a finite number of lines of known locations". Tomographic scanners estimate line integrals by sending an energy pulse of some type through an object and measuring how much energy is absorbed. The most important reconstruction algorithms are based on transform methods.

- Tactile sensing in Robotics - Because it represents an inexpensive and robust technology, tactile sensors have been studied $[26,31,34]$ as an alternative to computer vision for robotics. The robot touches the object several times, each time identifying a point on the boundary of it. From this data, the system seeks to determine what the object is.

- Theory of Learning - Abstractly, a concept is often modeled as a region in some highdimensional space $[6,9]$. Such a concept can be 'learned' by asking questions of the form "is this point in the region?," and developing an approximation of the region's shape. For efficient learning, it is important to ask questions in a way that provide as much information about the region as possible. Lower bounds are also of interest, for showing that it requires a large number of questions to approximate the region proves no efficient learning strategies exist.

Geometric probing considers problems of determining a geometric structure or some aspect of that structure from the results of a mathematical or physical measuring device, a probe. A variety of problems from robotics, medical instrumentation, mathematical optimization, integral and computational geometry, graph theory and other areas fit into this paradigm.

The problem of geometric probing was introduced by Cole and Yap [15] and inspired by work in robotics and tactile sensing [26, 72]. It has since been the focus of at least four dissertations [30, 50, 59, 74] and several additional papers. The dissertation [74] is the most complete presentation of results in probing, and is the basis for this paper. A comprehensive collection of open problems and the following taxonomy of probing problems appears in [75].

There are a wide variety of interesting models of sensors, with inspiration either from physical sensing devices or geometrical operations.

- Finger Probes - which measure the first point of intersection between a directed line and an object.

- Hyperplane Probes - which measure the first time when a hyperplane moving parallel to itself intersects an object.

- X-ray Probes - which measure the length of intersection between a line and an object.

- Half-space Probes - which return the area or volume of intersection between a half-space and an object.

- Cut-set Probes - which for a specified graph and partition of the vertices returns the size of the cut-set represented by the partition. 
More sophisticated sensing devices can be constructed from considering aggregates of probes sharing certain properties, such as the set of all probes which are parallel to a given line or which pass through a given point.

There are a variety of different properties which we are interested in optimizing or bounding:

- Determination - how many probes are necessary to completely determine a particular object? We are interested in both upper and lower bounds. Note that we use "determination" to refer to interactive reconstruction strategies, which Gardner [23] terms "successive determination".

- Verification - given a reputed description of the object, how many probes are necessary to test if the description is valid?

- Computational Complexity - the complexity of planning the probes can be measured under a RAM cost model, assuming that an oracle returns the results for a specified probe in unit time.

- Simulation - given a probe model and a representation of the object, how much time and space is necessary to simulate an actual probe?

- Feature Determination - how many probes are needed to determine some feature of the object, such as volume, orientation, or convexity?

Results in probing are most naturally ordered by probing model. To provide a better understanding of the field, the rest of this paper presents the major results for various models.

\section{$2 \quad$ Finger Probes}

Tactile sensing is an important paradigm in robotics. Cole and Yap [15] developed the notion of a finger probe to model tactile sensors used in robotics. A finger probe is defined to be the first point of intersection $p$ between a directed line $l$ and an object $P$. The term probe will sometimes be used to refer to $p$ and sometimes to $l$, and we rely on context to distinguish between these meanings.

The notion of finger probing has inspired work on a variety of problems and a growing body of literature $[8,11,16,30,53,55]$. We present the main results of this literature. Together, these problems show both the power and limitations of finger probing and thus of tactile sensing in robotics.

We assume that we are given $O$, a point within the interior of a convex polygon $P$. Without this information, we have no idea where $P$ is located, and an unbounded number of probes can be required to find it. From each probe, in addition to the contact point we obtain a half-line defined by $p$ and $l$ which does not intersect $P$. This and the convexity restrictions on $P$ can be used to identify points known to be within $P$ or known to be outside of $P$. Together, these two sets represent the state of our knowledge about $P$. Let inside $(P)$ 
Figure 1: Information from Finger Probing.

be the closed set of points which can be proved to be within $P$, specifically, they are on or within the convex hull of the set of contact points $X \cup$ o. Let outside $(P)$ be the set of points which can be proven not to lie on or within $P$. Specifically, $z \in$ outside $(P)$ if there exists a point $x \in X$ such that $x$ is in the interior of $\operatorname{conv}(\{z\} \cup X)$, where $\operatorname{conv}(S)$ is the convex hull of a set $S$. Figure 1 shows inside $(P)$ and outside $(P)$ for a specific collection of probes. We say that $P$ is determined when $\operatorname{inside}(P) \cup$ outside $(P)=E^{d}$, where $d$ is number of dimensions of $P$.

We emphasize that we are interested in absolute determination, not an approximation to $P$. When a convex polygon is determined we have identified the exact coordinates of each of the $n$ vertices. This notion of determination will be used throughout this paper, even for probing models such as x-rays which do not return absolute information.

One final subtlety concerns tangent probes. Cole and Yap's model [15], which will be primarily considered in this paper, has a finger probe along an edge of $P$ returning the first vertex it encounters, although [12] assumes such probes miss the polygon completely. It is even less clear what should be returned when the probe line intersects only a vertex of $P$, but [15] makes the assumption that such probes miss $P$ entirely. The point is moot as far as this paper is concerned, as we avoid the use of such probes.

\subsection{Determination and Verification}

The fundamental problems in geometric probing are determination and verification. Determination counts the number of probes necessary to reconstruct an object $P$. We note that 
the sequence of probing outcomes for a determination provides a complete representation for $P$, because the probing algorithm can be used to generate the probe specifications. Thus determination strategies suggest alternate representations for geometric objects.

Verification counts the number of probes necessary to prove that $P$ is indeed the object in question. This problem can be thought of as 'non-deterministic' probing, since it is assumed that the algorithm always makes the right probe for any $P$. To do this, it is assumed that the algorithm has a description of $P$ as its input. Verification problems are important because they provide a lower bound for determination, since $P$ is an input for verification.

Cole and Yap presented the following optimal $2 n$ probe strategy for verifying a convex $n$-gon. To show necessity, note that each vertex and edge must be probed at least once, since an unprobed vertex can be truncated and an unprobed edge extended with another vertex. For sufficiency, we note that three collinear points must all lie on the same edge of $P$, by convexity. Thus probing each vertex and edge interior once determines the extent and position of each edge of $P$.

The deterministic version of this problem is more difficult. The following strategy is a variation on the algorithm in [15].

Theorem $13 n$ finger probes are sufficient to determine a convex $n$-gon.

Proof: Our strategy will consist of two phases. The first phase ends when there are three probes that are incident upon the same edge $e_{1}$, the second when $P$ is determined.

All probes in the first phase will be directed through the origin $O$, and thus the $i$ th probe can be specified by either its angle $\theta_{i}$ or contact point $x_{i}$. Send three probes through $P$, where $\pi<\theta_{3}<\theta_{2}<\theta_{1}<2 \pi$. If $x_{1}, x_{2}$, and $x_{3}$ are collinear, we have completed the first phase. If not, note that $x_{1}$ and $x_{3}$ cannot be incident upon the same edge because of convexity constraints. We will insure that the next edge $e_{n}$ in a counter-clockwise direction from $e_{1}$ has at most one probe incident upon it at the end of the first phase.

Let $m$ be the slope of the line defined by $x_{2}$ and $x_{3}$. If $m$ is not between $\theta_{1}$ and $\theta_{2}$, as in Figure 2a, we will aim the fourth probe between $\theta_{1}$ and $\theta_{2}$, and then until three contact points are collinear aim probe $i+1$ with angle thet $a_{i+1}$ where $\theta_{2}<\theta_{i}<\theta_{i+1}<\theta_{1}$. The three collinear points defining $e_{1}$ will be either $x_{i-1}, x_{i}, x_{1}$ or $x_{2}, x_{4}, x_{1}$, or $x_{i-2}, x_{i-1}, x_{i}$. In the two cases, convexity prevents $\left(x_{2}, x_{3}\right)$ from being $e_{n}$, and in the third, we have shown that $x_{1}$ cannot be on the same edge as $x_{3}$. If $m$ is between $\theta_{1}$ and $\theta_{2}$, as in Figure $2 \mathrm{~b}$, we will aim the fourth probe between $\theta_{2}$ and $m$, and then until three contact points are collinear aim probe $i+1$ with angle thet $a_{i+1}$ where $\theta_{2}<\theta_{i}<\theta_{i+1}<m$. Edge $e_{1}$ will again be defined by $x_{i-1}, x_{i}, x_{1}$ or $x_{2}, x_{4}, x_{1}, x_{i-2}, x_{i-1}, x_{i}$ and the same arguments apply.

For the second phase of this strategy, we note that to determine our first vertex $v_{1}$, between $e_{1}$ and $e_{n}$, we can probe along the directed line from $x_{i}$ to $x_{i-1}$. After determining $v_{1}$, we proceed in a clockwise direction around $P$. If $e_{i}$ is the most recent verified edge, and $x_{a}$ and $x_{b}$ are the next two probe contact points around $P$, we aim the next probe along the line defined by $p$ and $O$, where $p$ is the intersection of $e_{i}$ and the line through $x_{a}$ and $x_{b}$ as in Figure 3. If the contact point is $p$, then $p$ is vertex $v_{i}$ of $P$ and a new edge is determined by $x_{a}$ and $x_{b}$. If not, the contact point lies on another edge. This process is repeated until $x_{a}$ is $v_{1}$ and $v_{1}, v_{n}$ and $x_{b}$ are collinear, so $P$ is completely determined. Since at most one 
Figure 2: Determining the first edge with $2 \mathrm{n}$ probes.

Figure 3: Determining the next edge of $\mathrm{P}$. 
Figure 4: Two distinct $n$-gons defined by $2 n-2$ points.

probe was incident upon this last edge $e_{n}$ from the first phase, and it is probed in the second phase only if necessary, it will be probed exactly once in its interior.

In summary, the interiors of $n-2$ edges are incident upon at most two probes each, the interior of $e_{1}$ is incident upon three probes, $e_{n}$ by one probe, and all $n$ vertices are verified once. Thus the number of probes used is at most $2(n-2)+3+1+n=3 n$.

The probe directed along $e_{1}$ would be very unreliable for a real world application, since only a slight perturbation could cause the probe to miss $P$ entirely. A more robust strategy would aim through the origin each time, and in this case $3 n+1$ probes are necessary and sufficient, will be discussed in Section 3.2.

Cole and Yap prove a lower bound of $3 n-1$ for determination, which raises to $3 n$ under the mild assumption that a probe which passes through one vertex of $P$ and not its interior has a contact point at infinity. This proof is surprisingly complicated, involving substantial case analysis. See [15] for details.

Figure 4 gives a simpler proof of the slightly weaker result that at least $3 n-1$ finger probes are necessary to determine a convex $n$-gon. We shall construct an adversary which will force at least $3 n-1$ probes from any probing strategy. Our adversary will use the following strategy: for as long as possible, all contact points will be on a circle around $O$. Also, no probe will contact a vertex until it is unavoidable. To delay these exceptions, we may relabel the contact points until it leads to a contradiction.

First note that for any $2 n-2$ points on a convex curve we can construct two convex $n$-gons such that each point is on the interior of an edge of each polygon, as is illustrated in Figure 4 . Thus the $2 n-1$ th probe can be forced not to contact a vertex by suitable choice of the two possibilities. To verify an $n$-gon, each of the $n$ vertices must be probed. Thus at least $2 n-1+n=3 n-1$ probes are necessary.

Unfortunately, the powers of finger probing prove sharply limited when we attempt to generalize the objects beyond a single convex polygon. Under Cole and Yap's model, there does not exist a finite strategy for determining a star-shaped polygon or multiple convex 
Figure 5: A star-shaped polygon which is unverifiable by finger probing.

polygons using finger probes [74]. For multiple objects we make our standard assumption that we know the coordinates of a point $O$ within each polygons, which provides the general location of each object.

To show that there does not exist a finite strategy to verify a star-shaped polygon with finger probes, even if the vertices are in general position, consider the star-shaped polygon in Figure 5. The difficulty is in verifying the edge $e=\left(v_{2}, v_{3}\right)$. No number of probes intersecting $e$ is sufficient, since a polygon $\bar{P}$ can be constructed for any such set of probes where the points of intersection are collinear despite each being incident upon different edges. Since $v_{1}$ and $v_{4}$ prevent probing directly along $e, P$ is not distinguished from $\bar{P}$. This proof does not hold when probes may originate from a finite distance, and using a different finger probing model to determine non-convex polygons is discussed in Section 2.3.

Dobkin, Edelsbrunner, and Yap [16] consider probing convex polytopes in higher dimensions. Beyond two dimensions, the number of vertices is no longer identical to the number of facets, so upper and lower bounds must be expressed in terms of $f_{i}(P)$, which represents the number of $i$-dimensional faces of $P$, for $0 \leq i \leq d$. So $f_{0}(P)$ is the number of vertices, $f_{1}(P)$ the number of edges, and $f_{d-1}(P)$ the number of facets, the $(d-1)$-dimensional faces, of $P$.

They show that $f_{0}(P)+5 f_{2}(P)$ finger probes are sufficient to determine a convex polytope in $E^{3}$. The basic strategy is similar to what we have seen in the plane; conjecture and verify vertices and facets of $P$. If we can insure that only a constant number of probes are incident upon each face, this yields a linear probe strategy. However, the generalization is not straightforward. In $E^{2}$, three collinear contact points verify an edge. Unfortunately, no number of co-planar contact points are sufficient to verify a facet in $E^{3}$, unless one point lies within the convex hull of the others, since these points can simply represent a cross-section of $P$.

Also in [16], a lower bound of $f_{0}(P)+f_{2}(P)$ is given for verification, noting that each 
vertex and facet must be probed to ensure all vertices have been confirmed. These results are also generalized to $d$ dimensions, for a lower bound of $f_{0}(P)+f_{d-1}(P)$ and an upper bound of $f_{0}(P)+(d+2) f_{d-1}(P)$. Lindenbaum and Bruckstein [51] give an improved lower bound of $d f_{0}(P)+f_{d-1}(P)$.

\section{$2.2 \quad$ Restricting the Objects}

In the strategies we have considered thus far, all that was known about the objects was a point within the interior and that they were convex. If we know more information about the object, we may be able to determine it with fewer probes. Sometimes the extra information reduces the number of probes required by only an additive constant, which becomes negligible as $n$ gets large. However, sometimes the multiplicative constant can be improved.

A natural question is how much knowing the number of vertices of $P$ helps in probing it. Cole and Yap considered this problem, showing that 8 probes are sufficient to determine a triangle and giving a lower bound of $2 n+1$ probes. An elementary $3 n-1$ probe strategy for determining a convex $n$-gon if $n$ is known is given in [74]. This modifies the stopping criteria of Cole and Yap's strategy to save a single probe.

Tightening these bounds is an open problem. The subtlety of a tight lower bound argument when $n$ is known is illustrated by the dramatic consequences for verification. $3\lceil n / 2\rceil$ probes are sufficient to verify a convex $n$-gon if $n$ is known [74]. Simply label the edges as even or odd. For each even edge $e$, make three probes: along $e$ in both directions and one incident to the center of $e$. These probes verify each even edge and prove that the endpoints are vertices. Clearly, knowledge of all $n$ vertices determines $P$.

Since constructing high-level computer vision systems has proven to be a difficult problem [42], tactile sensing has been studied as an alternative for robotic assembly systems. The abstraction of such systems are very similar to finger probing. In a mechanical assembly problem, the geometry of the parts is known to the robot, which must use sensing to determine their type and orientation. Such problems are called model-based and differ from the determination problems we have discussed in that the objects come from a finite set.

Here we review the work that has been done in tactile sensing and robotics. This work is closely tied to real world applications. The problem of determining an object via tactile sensing can be studied from several different perspectives. Researchers at MIT [26, 33] have considered using heuristics to reconstruct polyhedra from random or oblivious probes, instead of defining a strategy to plan the probes. Their probing model is somewhat more powerful than the finger probe, returning both a contact point and the surface normal at that point. From a small number of such tactile probes, they construct an interpretation tree consisting of the possible mappings between contact points and the faces of model polyhedra. By using local geometric constraints such as whether the distance between two probes is consistent with the edge labelings, they prune this interpretation tree for $m$ edges and $s$ probes from $m^{s}$ nodes to, what is in practice, a small number of interpretations. This system works well even with non-convex polyhedra and for modeling sensing errors. It has been extended, with somewhat less success, to the bin searching problem of overlapping parts [34] by adding a 'null face' to the interpretation tree, which accounts for probes which do not contact the 
intended object.

Grimson [31] made a brave attempt to explain the excellent average case performance of these oblivious tactile sensing strategies. If $p$ is the probability that two random probes represent a consistent set of interpretations, then it can be shown that the expected number of consistent interpretations $I_{\text {exp }}$ after $s$ probes is

$$
I_{\text {exp }}=m^{s} p^{\left(\begin{array}{l}
s \\
2
\end{array}\right)}
$$

where $m$ is the number of model faces. From this can be calculated the expected number of probes where $I_{\text {exp }}$ is largest, and when there remains only one consistent interpretation. One consistent interpretation can be expected when

$$
s=1-\frac{2 \log m}{\log p}
$$

Of course, it is difficult if not impossible to determine $p$ for a particular model. Regular convex polygons will never converge upon a single interpretation. Grimson computes $p$ by assuming a uniform distribution of points in his relative configuration space. His results compare well with the results of simulations and are generalized to account for uncertainties in measurement.

Several researchers have developed strategies for using these tactile sensors. Grimson [32] proposes the following scheme to select a probe which distinguishes between two or more possible orientations. Select a probing direction, ie. the slope but not the intercept. Project the visible vertices of the orientations onto the line normal to this direction. This divides the line into segments. The midpoint of the segment which distinguishes the largest number of orientations, subject to measurement uncertainty, defines the probe. An extensive analysis of the error terms are provided.

Ellis, Riseman, and Hanson [19] describe a similar system which represents probes incident upon an edge as a trapezoid in a projective space and selects a probe which is represented in the intersection of a number of trapezoids. They consider the problem of stability, where a probe at too oblique an angle deflects off the polygon. Spyridi and Requicha [80] consider inspection problems with coordinate measuring machines, which are closely related to finger probes.

For industrial robot applications in manufacturing and assembly, either the parts must be carefully positioned for the robot to manipulate or the robot must be able to detect the orientation of parts and adjust to them. Since the technical problems associated with computer vision systems remain very difficult, systems for the sensorless orientation of objects [60] or using simpler, more robust sensors to determine orientation have been studied. Natarajan [61] poses the problem of determining the orientation of a known polytope moving past simple ray sensors on a guide plane, with improved results appearing in [74]. Lyons and Rappaport [55] consider a similar problem: given a collection of $m$ known convex polygons with fixed orientation on a plane, how many finger probes are required to identify an instance of one of the models? They prove that $m-1$ finger probes are necessary and sufficient to 
Figure 6: Determining the next edge of model polygon $P$.

determine the instance, which is interesting because this result is independent of the number of sides in the models. This property is lost when the models may assume arbitrary orientations. Belleville [7] shows that the problem of minimizing the number of probes to identify a unimodal convex polygon from a set of oriented models is NP-hard, when all probes must be made simultaniously, instead of interactively as in the problem posed by Cole and Yap.

Since model based problems predominate in robotics, it is not surprising that general probing strategies have been developed. Two distinct model-based determination problems will be considered, the first when all the models are convex and the second with much more general objects. For both problems, we assume there are $m$ distinct models of at most $n$ sides each.

Bernstein [8] gives a solution to this problem for convex polygons. Surprisingly, his result that any convex polygon from a known set of models can be determined in $2 n+2$ probes, is independent of the number of models $m$. Joseph and Skiena [43] improved this to within an additive constant of optimality, showing that $n+3$ probes are sufficient and $n-1$ necessary to determine a convex $n$-gon $P$ selected from a finite set $\rho$.

The intuition behind these strategies is that if the set of candidate polygons is known, it is possible to aim probes close enough to each other to insure that they are incident upon the same edge. In particular, we need to pre-calculate two quantities from the set of polygons $\rho$.

The first quantity is an angle $\theta_{\min }^{\rho}$ small enough, so whatever the point is selected as origin and whatever polygon is the model, aiming five probes at $O$ within $\theta_{\min }^{\rho}$ means the contact points are on at most two consecutive edges. Thus one edge gets at least three contact points, determining it.

For any convex polygon $P$ with vertices $V=v_{1}, \ldots, v_{n}$, let $h_{\min }^{P}=\min \left\{d\left(\overline{v_{i} v_{i+1}}, v\right) \mid v \neq\right.$ $v_{i}, v_{i+1}$, where $d(l, v)$ represents the minimum distance from line $l$ to point $v$. Thus any probe sent parallel to a known edge $e$ of $P$ within $h_{\min }^{P}$ of $e$ is guaranteed to hit the next edge, as in Figure 6. Finally, let $h_{\min }^{\rho}=\min \left\{h_{\min }^{P} \mid P \in \rho\right\}$.

With these two quantities pre-calculated for $\rho$, a strategy is to send probes towards $O$ within an angular sector of $\theta_{\min }^{\rho}$ until three are collinear. This must happen after at most five probes regardless of which model $P$ is, with up to two probes contacting a neighboring edge. We now walk around $P$, aiming two probes parallel to the previous edge of $P$ within $h_{\text {min }}^{\rho}$ of it, so these two probes are guaranteed to contact the next edge. These two points determine the new edge and we can resume walking around $P$ in this manner until $P$ is 
determined. By refining $h_{\min }^{\rho}$ as discussed in [43], in fact one probe is sufficient to determine the next edge, resulting in an $n+3$ probe strategy. The key idea is aiming the probes below the lowest point of intersection in an arrangement of superimposed models.

Things get much more difficult when the models move beyond convex polygons. For example, $\theta_{\min }^{P}$ is no longer necessarily $>0$, since a point within $P$ can lie on a line defining one of its edges. Unfortunately, as we move beyond convex polygons, we lose the independence on $m$. In [74], it is shown that $O(m n)$ probes are both necessary and sufficient to determine an $n$-gon $P$ selected from a set $\rho$ of $m$ star-shaped models if $O$ is within the kernal of $P$.

This dependence on $m$ proves it is difficult in the worst case to distinguish between multiple non-convex models. Thus, it is understandable that heuristics have been used for this problem, particularly in light of Grimson's [32] average case results.

\subsection{Other Models of Finger Probes}

We have considered using finger probes to determine an unknown convex polygon or identify a polygon drawn from a known set of objects. Researchers have also studied different models of tactile sensors, with interesting questions of how increasing the power of the probe effects its efficiency at determination and verification. The goals of such efforts include increasing robustness, determining non-convex objects, and studying the effect of parallelism.

Dobkin, Edelsbrunner, and Yap [16] consider the problem of probing with uncertainty. This is clearly of practical importance, since any real world sensing device would have some degree of measurement error, and the probing strategies we have seen are not robust. For example, we aim probes directly at vertices, which is not a stable operation. One way to formalize uncertainty is to assume a lower bound $l$ on the size of any edge of $P$ and that the returned contact point on all probes is within a distance $\epsilon$ of the actual contact point. If $\epsilon$ is a function of $l$, meaningful results can be expressed in terms of $\epsilon$ and $n$. Particularly interesting will be the relationships between the uncertainty of the probing device $\epsilon$, the desired accuracy of reconstruction $\delta$, and the number of probes needed to achieve it. Obviously, there are many alternative ways to formulate probing with uncertainty. Lindenbaum and Bruckstein [52] remove the assumption that the object is a polygon, and provide an adaptive strategy to find an approximate reconstruction under the Hausdorff metric. This work is based on line probes, which are duals of finger probes as discussed in Section 3.1. Related work on polygon approximation appears in [65, 70].

As we have seen, Cole and Yap's finger probing model is not powerful enough to determine non-convex objects. There are three major reasons for this, illustrated in Figure 7. A tiny crack in an edge can go forever undetected, since no finite strategy can explore the entire surface of the polygon. Second, it is easy to construct non-convex polygons whose features cannot be entirely contacted with straight-line probes. Finally, there exists no constant $k$ such that $k$ collinear probes determines an edge.

Alevizos, Boissonnat, and Yvinec [4,3] attack each of these problems to develop a tactile sensing model which can determine a large class of polygons. To eliminate the problem of tiny cracks, they limit their attention to polygons where each of the supporting lines of the edges are unique. To eliminate the problem of reachability, they generalize the probe 
Figure 7: Generalizing finger probing to non-convex objects.

paths from directed lines to arbitrary curves. Finally, they augment the sensor to return the surface normal as well as the contact point. This is a reasonable assumption since the surface normal is determined locally, and permits an edge to be determined with each probe. In the event that a probe contacts a vertex of the polygon, they assume that normals for both adjacent edges are returned.

Under this model, they prove that $3 n-3$ probes are necessary and sufficient to determine a simple $n$-gon with non-collinear edges. Similar questions can be asked for collections of $k$ polygons or polyhedra in $E^{3}$. Boissonnat and Yvinec [11] (corrected [12]) prove that a scene of $k$ polyhedra with a total of $n$ faces and $m$ edges can be determined in $10 n(m+k) / 3-2 m-3 k$ probes, where no two edges are collinear and no two faces coplanar.

The relaxation of the convexity constraint has a variety of effects on reconstruction from finger probes. Consider probing the boundary of a simple, closed curve. With convex objects, the order in which a set of contact points occur on the curve is uniquely determined by their convex hull, but the situation gets more complicated with arbitrary closed curves. Alevizos, Boissonnat, and Yvinec [2] prove that a unique solution exists given the probe paths as well as the contact points for arbitrary closed curves, and further such an order can be found in $O(n \log n)$ time where $n$ is the number of probes.

Parallelism is a technique commonly employed to speed up a computation. The problem associated with parallelism is the degree of achievable time speedup for a problem of size $n$ given $k$ processors. Clearly, in the best case, the job can be completed $k$ times faster. However, the structure of most problems makes it impossible to realize this. This is true in real life as well as theory, as anyone who has ever been on a committee can attest. 
Now we can consider the problem of to what extent we can speed up the number of "rounds" it takes to determine a convex object, when we have the ability to make up to $k$ probes per round. This is similar in flavor to the "sorting in rounds" problem [64] which has been extensively studied. By Cole and Yap's strategy, $3 n$ probes suffice for any $k \geq 1$, and their lower bound shows $3 n / k$ rounds are necessary. For $k=2$, [74] shows that $8 n / 3$ probes are sufficient.

Lindenbaum and Bruckstein [53] prove bounds tight to within an additive constant for all $k$. For $k=2$, they show $2 n-2$ probes are necessary and $2 n$ sufficient. For $k=3$, they show $2 n-3$ probes are necessary, and $2 n$ sufficiency follows from the strategy for $k=2$. For $k=4$ or $k=5$, they show that $(4 n-5) / 3$ are necessary and $\lfloor(4 n+2) / 3\rfloor$ sufficient. Finally, for $k \geq 6 n$ probes are necessary and $n+1$ sufficient. Their parallel strategies are fairly simple to implement and describe, but the complexity analysis is more difficult.

\section{$3 \quad$ Hyperplane Probes}

At an early age, we are taught that it is not polite to point. Finger probes are defined in terms of a moving point, like a finger. Here we introduce the notion of the hyperplane probe, which is defined as a moving hyperplane which stops when it is tangent to the polytope. The palm of a hand which hits an object is an example of such a probe. As we shall see, this anthropomorphic description is not the only connection between finger and hyperplane probes.

The terminology has the potential to become confusing, as the same concept has been referred to in the literature as line probes [49], support planes [30, 51], and hyperplane probes [16]. We will use the following terminology. A hyperplane probe will be a $(d-1)$-dimensional hyperplane, typically aimed at a $d$-dimensional object. A one-dimensional hyperplane probe will be called a line probe. Like finger probes, hyperplane probes originate from infinity.

\subsection{Duality between Finger and Hyperplane Probes}

Duality [14] is one of the fundamental ideas of computational geometry and occurs frequently in many areas of mathematics. Two problems $P_{1}$ and $P_{2}$ are duals if there is a transform which relates each instance of $P_{1}$ to a unique instance of $P_{2}$ and each instance of $P_{2}$ to a unique instance of $P_{1}$. Thus any algorithm which solves one of the problems can also be used to solve the other, since any instance of $P_{1}$ can be transformed to one of $P_{2}$ and solved with the known algorithm. Duality is important because it provides alternative representations for problems which lead to solutions which otherwise might not become apparent.

We will demonstrate the power of duality by proving that finger and hyperplane probing are really identical problems. This duality relationship was independently discovered by Dobkin, Edelsbrunner, and Yap [16] and Greschak [30].

Let $P$ be a convex polytope and $O$ be a point within $P$. Each point $p \neq O$ defines a vector in $E^{d}$. Let the dual of $p, d(p)$, be the closed half-space containing $O$, defined by the hyperplane normal to the vector $p$ containing the point $p /|p|^{2}$. The $d u a l$ of the polytope $P$ 
Figure 8: The dual of a polygon.

is defined

$$
D(P)=\bigcap_{p \in P} d(p)
$$

Figure 8 shows the effect of the duality transform on a polygon. Each vertex $v$ of $P$ is replaced by an edge $e$ of $D(P)$ which is normal to $(O, v)$. Likewise, the normal to each edge of $P$ defines a vertex of $D(P)$. The dual of any hyperplane probe which contacts $v$ of $P$ corresponds to a finger probe which intersects $e$. If we consider a finger probe moving towards the origin, it dualizes to a hyperplane probe moving normal to it away from the origin. The finger probe contacts $P$ at the last point in time when the hyperplane probe intersects $D(P)$. We can thus view the hyperplane as moving towards $D(P)$ and stopping when it touches $D(P)$ - this gives the same result and is more intuitive than the hyperplane that moves away from the origin.

Thus, any strategy for finger probing where all probes are aimed through $O$ defines a strategy for hyperplane probes. Dualizable results include the $2 n$ bound for verification of convex polygons and and the $3 n$ determination lower bound. Unfortunately, the optimal $3 n$ determination strategy of Theorem 1 and the $n+3$ model-based strategy of [43] rely on at least one non-origin probe and do not dualize to line probes.

In fact, the line probing model is slightly weaker for determining convex polygons than the finger probing model. Li [49] showed $3 n+1$ line probes are necessary and sufficient for determination, using a simplified dual of the finger probing strategy of Theorem 1 .

If we dualize a finger probe $f$ which is not directed through $O$, it sweeps out a region between two hyperplanes, as the hyperplanes rotate about an axis. The first hyperplane is defined by its normal $f$ and $O$ and the second hyperplane intersects $D(P)$ in the appropriate point. Thus any finger probing strategy is equivalent to a combined strategy of hyperplane 
probes and probes which rotate hyperplanes around a $(d-2)$-dimensional flat, which we call supporting hyperplane probes. The $(d-2)$-dimensional axis of rotation is a dual of the line defining the finger probe. The direction of rotation is specified by the direction traveled along $f$.

Supporting hyperplane probes occur naturally in various robotics problems. In $E^{2}$, we can consider supporting line probes. Specified by a starting point $p$, angle, and direction of rotation, they return the angle of the line through $p$ which first intersects $P$. By considering two such probes with opposing directions of rotation, we obtain an angle range over which $P$ lies. This is exactly the information obtained by gripping the object between two fingers of a rigid but endless hand. An alternate and more realistic problem involves a finite sized claw, such as the section of an object one can grab with a hand. Okada and Tsuchiya [62] discuss a system which distinguishes between a ball, cylinder, and various prisms using the contact points of finger positions while grasping the object.

The model-based finger probing strategies of [8] and [43] do not dualize to line probes. However, in [43] we prove that $2 n+4$ probes are sufficient to identify a convex $n$-gon from a collection of known models $\rho$. Further, $2 n-3$ probes are necessary, so the bounds are tight to within an additive constant.

\subsection{Silhouette Probes}

One problem researchers in computer vision must contend with is that photographic images can provide too much information. It is difficult for a program to associate regions of different shadings as portions of the same object. Thus they often deal with silhouettes, thresholded binary images when the object is dark and the rest of the image is light, like a shadow where the illumination originates from infinity. Projecting a silhouette defines an infinite cylinder in which the object must lie. Intersecting several silhouettes refines our knowledge of the object's shape and has formed the basis of many vision and solid modeling systems. For example, Wang, Magee, and Aggarwal [86] describe a system which performs model based recognition of different types of cars from their silhouettes. See Martin and Aggarwal [58] for more extensive references on the applications of silhouettes.

There is a duality relationship between silhouette probes and the equally natural notion of cross-sectional slices. The cylinder defined by a silhouette with direction $\bar{v}$ represents the intersection of half-spaces containing $O$ and defined by all hyperplane probes perpendicular to $\bar{v}$. The duals of these hyperplanes are the complete set of finger probes along a slice through $O$ perpendicular to $\bar{v}$.

In two dimensions, a silhouette probe represents two line probes with identical slope originating from $\pm \infty$. Each such probe dualizes to the pair of finger probes defined by a line through $O$, the cross-sectional interpretation. Li [49] terms two-dimensional silhouette probes projection probes and proves that $3 n-2$ probes are necessary and sufficient to determine convex polygons with silhouettes. Thus in the worst case, only three probes are saved using projections instead of single line probes. Weaker results for this problem were independently proven by [59]. Prince and Willsky [65] reconstruct convex sets from noisy silhouette probes, also called supporting lines. 
Li proves the upper bound by describing three projection probes which do the work of the first six line probes in the $3 n+1$ determination strategy. From here the $3 n+1$ strategy can be continued, with the other side of the projection probe ignored. To prove a matching $3 n-2$ lower bound, the adversary works with a convex polygon $P$ such that both the interior angles incident upon an edge $e$ are acute. Thus at least one half of any projection probe contacts $e$, meaning that once $e$ is determined half of each projection probe is wasted.

It is interesting that doubling the number of probes only buys us an improvement of three in the time it takes to determine polygons. Lindenbaum and Bruckstein's results reported in Section 2.3 show that more dramatic improvements are possible when the two probes per iteration are not restricted to the same line.

Silhouette probes also provide surprisingly little power in higher dimensions. Recall that silhouette probes are dual to cross-sectional slices. Dobkin, Edelsbrunner, and Yap [16] prove that $f_{0}(P) / 2$ cross-section probes $\left(f_{2}(P) / 2\right.$ silhouette probes) are necessary and $f_{0}(P)+5 f_{2}(P)\left(5 f_{0}(P)+f_{2}(P)\right.$ silhouettes $)$ are sufficient to determine a convex polyhedron in $E^{3}$. These bounds are optimal within a multiplicative constant, since $3 f_{0}(P)-6 \leq f_{2}(P)$ by Euler's formula.

A simple verification argument suffices to show the lower bound. Let $P$ have no three vertices be co-planar with $O$. Since every vertex must be the vertex of a cross-section to be verified, at least $f_{0}(P) / 2$ probes are necessary. For sufficiency, we note that a finger probe can be wastefully simulated by a cross-section probe perpendicular to the desired probing direction. The result follows from the complexity of the higher dimensional finger probing strategy of [16]. For silhouettes, we simulate a hyperplane probe.

It is an open problem to tighten these results, noting that Chazelle, Edelsbrunner, and Guibas [13] prove there exist polytopes such that each silhouette has at most $O(\log n / \log \log n)$ edges.

In the problems we have studied thus far, the dimension of the probe was always one less than the object being probed. For example, we used line probes to determine convex polygons. It is possible to develop a strategy for using line probes to determine convex polyhedra. This increased gap in dimension leads to interesting problems.

Edelsbrunner, Dobkin, and Yap [16] present a $9 f_{0}(P)+f_{2}(P)$ strategy for reconstruction with line probes. Unfortunately, their strategy is very unstable, as each vertex is verified by two opposing line probes, which sweep out a plane except for clipping the vertex. A more stable linear algorithm for determination with line probes appears in [74], where it is shown that $f_{0}(P)+f_{2}(P)$ line probes are necessary and $2 f_{0}(P)+f_{1}(P)+13 f_{2}(P)$ line probes sufficient to reconstruct a convex polytope $P$. Boissonnat and Yvinec [12] consider line probes to determine non-convex polyhedra.

\section{X-ray probes}

In this section, we consider the problem of reconstruction with x-ray probes. An $x$-ray probe $X(P, l)$ is defined to be the length of intersection between a polygon $P$ and the line $l$. X-ray probes have characteristics which make them more difficult to use than finger or hyperplane 
Figure 9: Different polygons satisfying the same collection of x-ray probes.

probes, but also have a close connection to important problems in tomography.

A collection of x-ray probes through an object provides us with a great deal of information about it but not directly with the coordinates of a point on the surface. Obtaining such absolute information is the difficulty in working with x-ray probes. Figure 9 demonstrates some of these difficulties; the collection of probes provides very little constraint on the location, shape, or number of sides of $P$. Another polygon $\bar{P}$ bears little resemblance to $P$, but gives identical results for the collection of probes. In fact, the complete set of probes $X(P, l(O, \theta))$ through $O$ over all angles $\theta$ describes two different polygons, $P$ and $P$ reflected through $O$, denoted as $-P$. Thus x-ray probes are a fundamentally different type of device from finger probes, although it is not clear which should be considered more powerful.

Radon [66] proved that any reasonable function $f(x, y)$ in two variables can be reconstructed from the complete set of line integrals of this function and developed the Radon inversion formula to specify how this can be done. This is the fundamental result upon which tomography is based. However, it can be shown [38] that in general all line integrals are necessary for reconstruction. We shall show that if $f(x, y)$ is restricted to convex polygons that a finite collection of $\mathrm{x}$-rays are sufficient for determination.

\subsection{Tomographic Reconstruction}

Tomography, from the Greek word for "cut", is the field which studies image reconstruction from projections. CAT scanners and other tomographic imaging systems represent a tremendous step forward in our ability to diagnose tumors and other medical problems. G. N. Hounsfield and A. M. Cormack were awarded the 1979 Nobel Prize in Medicine for their contributions to tomography. For more extensive surveys of tomography, see the textbook by Herman [38], a special IEEE Proceedings issue devoted to computerized tomography [39], 
or one of the following excellent articles [73, 79].

Tomographic scanners estimate line integrals by sending an energy pulse of some type through the object and measuring how much energy is absorbed. In a heterogeneous material, each different component will transmit a different percentage of energy per unit distance. The image reconstructed will be a map of these attenuation coefficients. A wide variety of sensing technologies have arisen, based on different types of energy sources.

The most common form is x-ray tomography, where a point radiation source is aimed through the patient to a point detector. The pattern used for collecting line integrals depends upon the type of scanner. In fan beam scanners, a collection of rays eminate from a single point source. Pencil beam scanners use several sources and detectors defining a set of parallel lines. In both cases, the sources and the detectors rotate together and measurements are taken at regular angular intervals. Single-photon emission computed tomography [45] relies upon the patient ingesting some radioisotope, with the sensors being located outside the body. A collimator is used to insure that the measured photons are identified by direction. Ultrasound [29] is a non-electromagnetic technology which uses sound as the energy source. Since sound needs matter to travel though, there are strong interactions with physical properties of the tissue to measure. Nuclear magnetic resonance [40] or NMR immerses patients in a magnetic field and measures variations in the field resulting from molecular interactions. These interactions depend upon the molecular properties of the material.

We have made the assumption throughout this paper there is no error associated with the results of any probe. While this abstraction makes possible all of our results, it is not a valid assumption for real measurements. For this reason, tomographic reconstruction algorithms rely heavily on mathematical techniques developed in signal processing to handle noise. It is these considerations that makes tomographic reconstruction techniques an interesting combination of pure mathematics and ad hoc engineering.

There are three major types of reconstruction algorithms in use. Backprojection methods reconstruct the value of a pixel as the average value of the rays through the point, normalized by the distance the ray passed through the pixel area. Obviously, such techniques cannot reconstruct a low density area surrounded by high density material, such as brain tissue surrounded by the skull. Although simple to implement and understand, they have no real mathematical pedigree and give poor results.

The most important reconstruction algorithms are transform methods, which are direct implementations of the Radon inversion formula. A detailed discussion of such methods is well beyond the scope of this paper. Since the theory is based on having the complete set of line integrals, interpolation techniques are used to approximate this from the set of observations. There is a close relationship between Radon and Fourier transforms which is exploited in these algorithms.

Since the final image to be displayed will be a matrix of pixel values, algebraic reconstruction techniques view reconstruction as an optimization problem, where each pixel is a different variable. Each probe represents a linear constraint, where the weighted sum of the pixels traversed by the probe is set equal to the value of the line integral. Due to insufficient data or errors leading to inconsistent constraints, this is typically solved as an optimization problem, according to some criteria such as least squares. Additional constraints to produce 
certain smoothness properties can also be included.

Three dimensional reconstruction is typically done by combining information from a number of two-dimensional slices. Boissonnat [10] describes an algorithm for constructing a polyhedron from a set of planar cross-sections based on Delaunay triangulations. There has been work done on full three-dimensional reconstruction by using non-parallel cross sections, although for many medical imaging applications obtaining such measurements is impossible. Such algorithms can be based on either transform methods or algebraic reconstruction techniques.

The probing problems in this paper are primarily interactive. The direction of probe $p_{i+1}$ is a function of the results of the first $i$ probes. This is different than in traditional tomographic systems, where the selection of probes is made in advance. To minimize the amount of radiation the patient is exposed to, it would be desirable to identify the probing direction which will provide the most information. With the advent of more powerful computers and special purpose signal processors, interactive tomography becomes a possibility.

\subsection{Bounds for X-ray Probes}

We can prove a non-trivial lower bound for determining convex polygons by x-ray probing with a comparison to finger probing, since any x-ray probe $X(P, l)$ can be simulated by two finger probes. Simply send finger probes down each end of the line, and compute and return the Euclidean distance between these two points.

Using Cole and Yap's $3 n$ lower bound for finger probing, this gives a $3 n / 2$ lower bound for determination with x-ray probes. However, Lindenbaum and Bruckstein [54] prove a $3 n-3$ lower bound for determination where two opposing finger probes on a line count as a single probe. This is a generalization of Li's [49] projection probe where the lines do not have to go through the origin. Since an x-ray probe provides strictly less information than one of these probes, $3 n-3$ x-ray probes are necessary to determine a convex $n$-gon.

For a determination strategy, we must obtain absolute information about the polygon. To obtain absolute information about $P$ from x-ray probes, it is necessary to think in terms of groups of probes which work together to determine something about $P$. This leads us to consider four different classes of probes, and study the powers and limitations they possess and how they interact to lead to probing strategies.

The first class of probes are origin probes, a set of x-ray probes all aimed through a common point $O$ within the object. Any x-ray probe which hits a convex polygon and avoids its vertices will go through exactly two edges of the object. The largest number of such edge pairs is $n$ [17].

We can define a mapping of $X(P, l(O, \theta))$ to two points $p_{1}$ and $p_{2}$ on $l(O, \theta)$ at a distance $X(P, l(O, \theta))$ from $O$, where $l(O, \theta)$ is the line through $O$ that encloses an angle of $\theta$ radians with the $x$-axis. By the following result, these points lie on hyperbolas defined by the edges probed through:

Lemma 2 Let $l_{1}: y=m_{1} x+b_{1}$ and $l_{2}: y=m_{2} x+b_{2}$ be two distinct lines and map each angle $\theta$ to the two points on line $l(O, \theta)$ at distance $d$ from $O$, where $d$ is the distance between 
Figure 10: Hyperbolas associated with two straight lines.

$l_{1} \cap l(O, \theta)$ and $l_{2} \cap l(O, \theta)$. Then these points define the hyperbolas

$$
y^{2}-x y\left(m_{1}+m_{2}\right) \pm y\left(b_{1}-b_{2}\right)-x^{2}\left(m_{1} m_{2}\right) \pm x\left(m_{1} b_{2}-m_{2} b_{1}\right)=0 .
$$

Proof: Consider the situation in Figure 10. The line $l(O, \theta)$ contains points $(x, y)$ such that $y=\tan (\theta) x$. With $t=\tan (\theta)$, we have

$$
d=\sqrt{\left(1+t^{2}\right)\left(b_{2} /\left(t-m_{2}\right)-b_{1} /\left(t-m_{1}\right)\right)^{2}} .
$$

The $x$-coordinates of the corresponding points can be found by subtracting the $x$-coordinates $x_{1}$ and $x_{2}$ of the intersections of $l(O, \theta)$ with $l_{1}$ and with $l_{2}$, respectively:

$$
x= \pm\left(x_{2}-x_{1}\right)= \pm\left(b_{2} /\left(t-m_{2}\right)-b_{1} /\left(t-m_{1}\right)\right) .
$$

This can be solved for $t$ and used with $y=t x$ to get $y$ as a function of $x$. To obtain a simpler formula, we set $A=m_{1}+m_{2}, B=m_{1} m_{2}, C=b_{1}-b_{2}$, and $D=m_{1} b_{2}-m_{2} b_{1}$. Then we have

$$
y=\frac{A x \mp C+\sqrt{(A x \mp C)^{2}-\left(4 B x^{2} \pm 4 D x\right)}}{2}
$$

and

$$
y=\frac{A x \mp C-\sqrt{(A x \mp C)^{2}-\left(4 B x^{2} \pm 4 D x\right)}}{2} .
$$

After simplification, this reduces to the assertion. 
Figure 11: The "spider web" $S(P)$ around a convex polygon $P$.

We will use Lemma 2 to determine the equations of the lines that contain edge pairs. If we have some number of origin probes through a common edge pair, then we can determine the hyperbolas through the associated points. From the equations of these hyperbolas, we then deduce the equations of the lines.

The hyperbolas that show up in Lemma 2 are defined by four constants $A=m_{1}+m_{2}$, $B=m_{1} m_{2}, C=b_{1}-b_{2}$, and $D=m_{1} b_{2}-m_{2} b_{1}$. It follows that, in general, four probes through a pair of edges is enough to determine the hyperbolas, and from the hyperbolas the equations of the lines that contain the two edges. Given $A, B, C$, and $D$, we have

$$
\begin{gathered}
m_{1}=\frac{A \pm \sqrt{A^{2}-4 B}}{2}, \quad m_{2}=\frac{A \mp \sqrt{A^{2}-4 B}}{2} \\
b_{1}=\frac{C}{2} \pm \frac{2 D+A C}{2 \sqrt{A^{2}-4 B}}, \quad \text { and } \quad b_{2}=\frac{-C}{2} \pm \frac{2 D+A C}{2 \sqrt{A^{2}-4 B}} .
\end{gathered}
$$

From these equations two limitations on our ability to reconstruct the edges are apparent. First, there is the ambiguity between $P$ and $-P$. More serious is that $b_{1}$ and $b_{2}$ are undefined when the square root vanishes, that is, when $m_{1}=m_{2}$. Thus any probing strategy using origin probes must take special action on parallel edges. Note, however, that there is no ambiguity between $P$ and $-P$ for parallel edges, since reflection through $O$ is equivalent to rotation through $\pi$ radians.

We can now recognize the structure formed by mapping origin probes to points. Each opposing pair of edges gives rise to their own pair of hyperbolas. Two adjacent hyperbolas meet on the line through $O$ and each vertex. Thus the probes define the extremes of a "spider web" $S(P)$ (see Figure 11) around the object. This permits us to interpret an origin probe for $P$ to be a finger probe on $S(P)$. Both $P$ and $-P$ generate the same spider web, $S(P)=S(-P)$. $S(P)$ consists of at most $2 n$ pieces of hyperbolas.

However, to make use of these properties of origin probes for a probing strategy we need some means to verify that four or possibly some higher but constant number of origin probes pass through the same pair of edges. With finger probes, it was possible to confirm an edge 
Figure 12: The histogram $C(P, \theta), \theta=0$, of a convex polygon $P$.

with three probes; two to define it and one in-between to verify it. Unfortunately, a constant number of extra confirmation probes lying on the same hyperbola is not sufficient to verify an edge pair [17].

Verifying edge pairs is the motivation for parallel probes. Parallel probes are a set of x-ray probes aimed with a common angle $\theta$. A complete collection of parallel probes for a given angle produce a histogram $C(P, \theta)$ (see Figure 12) of the thickness of the obstacle. This is the situation in a medical x-ray photograph. Formally, $C(P, \theta)$ is obtained as follows. Let $b$, the base line, be normal to the probing direction $\theta$ and call one of the half-planes defined by $b$ its positive side. Let $l(b, p)$ be the line normal to $b$ such that $p=b \cap l(b, p)$; thus, $l(b, p)$ has angle $\theta$. We map the probe $X(P, l(b, p))$ to the point on $l(b, p)$ at distance $X(P, l(b, p))$ from $b$ that lies in the positive side of $b . C(P, \theta)$ is the polygon bounded by $b$ and the images of all probes with angle $\theta$. For a convex $n$-gon this histogram will be bounded by up to $n$ line segments, including the one on $b . C(P, \theta)$ is convex for all convex $P$ over all angles $\theta$. Note that an x-ray probe with angle $\theta$ can be interpreted as a finger probe on $C(P, \theta)$. Each vertex of $C(P, \theta)$ determines a line on which must lie a vertex of $P$. Thus they provide a capability for verifying edge pairs. In fact, three parallel probes through a pair of edges are sufficient to verify them.

There are two apparent weaknesses of parallel probes. First, a finite number of them cannot be guaranteed to locate certain vertices, specifically the extreme vertices that correspond to the vertices of $C(P, \theta)$ that lie on the base line $b$. Without any bounding information, repeated probes may intersect the same pair of edges. Second, once a vertex is finally located, it is impossible without more information to distinguish whether there are one or two vertices on the line.

It is interesting to consider using an infinite number of parallel probes, as approximated in medical x-ray photographs where all probes perpendicular to the photographic plate are recorded at once. The first weakness vanishes although the second remains. This problem was first posed for convex sets by P.C. Hammer in 1963 [36] and will be discussed in Section 4.3 . 
Since parallel and origin probes have complementary properties of verification and identification, by combining them we can obtain our first piece of absolute information about the polygon. The idea behind this strategy is to bound a section of the polygon where we know there must be at least one edge pair and repeatedly send parallel probes to this section until three images on the boundary of the corresponding histogram are collinear. Once we have an edge pair, origin probes can be used to determine the lines containing the two edges. The procedure is complicated by parallel edges, but in [17] we show that $2 n+23$ x-ray probes are sufficient to identify the lines that contain the first pair of edges and a point on one of the two edges.

A complete probing strategy for $P$ could perhaps be constructed along these lines by repeating the process for each pair of edges. However, since $\mathrm{O}(n)$ edge pairs can be parallel this would lead to a quadratic number of probes. A simpler strategy can be developed once we know a point on the boundary of $P$.

The power of the finger probe is that it returns a point on the boundary of the polygon. To get a similar effect, we define the boundary probe, which relies on the observation that sending an x-ray line probe through a known point on the boundary of a convex polygon identifies another point on the boundary of the polygon. This means that once we have identified the coordinates of any point $p$ on the boundary of the polygon, any x-ray probe through $p$ determines another boundary point. The dual of Li's [49] line probe determination strategy can be modified to use boundary probes in place of finger probes.

Starting from one of the semi-verified edges, we will walk along the polygon, conjecturing vertices based on the intersection of the semi-verified edge and the line defined by the next two known points. Each of the $n$ vertices will eventually be probed, and each of the $n-2$ other edges will have at most two interior points probed, for a total of $5 n+19$.

For determination in higher dimensions, at most $f_{0}(P)+4 f_{2}(P)+(d+2) f_{d}-1(P)+46$ x-ray probes are required to determine a convex polytope $P$ in $E^{d}$ [17]. A linear lower bound in $E^{3}$ and higher dimensions can be obtained from the lower bound for finger probing and the fact that two finger probes can simulate an x-ray probe.

In any verification strategy, clearly each vertex and edge must be confirmed. Otherwise, $P$ could have a triangle on any unconfirmed edge or be truncated before any unconfirmed vertex. Since an x-ray probe passes through members of the set of edges and vertices, and there are at least $2 n$ points of interest, $n$ probes is a verification lower bound.

To verify a convex $n$-gon $P,(3 n / 2)+6$ x-ray probes are sufficient. Given the polygon to verify, three parallel probes are sufficient to verify the existence of a non-parallel edge pair and three origin probes are enough to define the hyperbola of it. One final probe to verify that $P$ is not reflected through $O$ identifies a boundary point.

From this boundary point, $n$ boundary probes can verify the vertices. The remaining $n-2$ edges can be verified with $(n-2) / 2$ probes, each bisecting a different pair of edges. Since $P$ is the convex hull of its vertices, none of these probes can have length other than expected without violating convexity unless there exists another vertex. Since $2 n$ finger probes are required to verify polygons, this is a case where x-ray probes are more powerful than finger probes. 


\subsection{Hammer's X-ray Problem}

P. C. Hammer [36] posed the following problems in 1963: How many x-ray pictures must be taken to permit exact reconstruction of a convex body if the x-rays issue from a finite point source? How many are needed if the x-rays are assumed to be parallel? These problems have since generated a substantial literature $[20,21,22,24,27,67,85]$ which is based on integral geometry [71]. The distinction between the two problems is basically the distinction between origin and parallel probing [17] models as discussed above.

In the terminology associated with Hammer's problem [23], a class of sets is "determined" by $n$ directions if there are $n$ predetermined directions such that all sets can be reconstructed from these $n$ projections. Further, a class of sets is "verified" by $n$ directions if for each particular set there are $n$ projections which distinguish this set from any other. Note that this definition of determination is different from the notion of interactive reconstruction used previously in this paper. Giering [27] proved that three parallel x-rays are sufficient to verify any convex set, where a parallel x-rays consists of the set of all x-ray probes parallel to a given direction. For the purposes of x-ray problems, a parallel x-ray can be reguarded as equivallent to the corresponding Steiner symmetral. Gardner [22] and Volčič [84] present simpler proofs of Giering's result. Gardner and McMullen [24] showed that any four parallel $\mathrm{x}$-rays are sufficient to determine a convex set, so long as their directions are not a subset of the directions of diagonals of an affinely regular polygon. There also exist a body of results for point sources, the complete set of x-ray probes originating from a point $p$, which may lie either inside or outside the convex body. This is a more powerful probe than the complete set of x-ray probes passing through $O$ (an origin probe), since the second probe would be unable to distinguish between a convex set $K$ and the same set rotated $\pi$ radians around $O$. Falconer [21] proved that two point sources $p_{1}$ and $p_{2}$, which lie on a line through the interior of $K$ are sufficient to reconstruct $K$. Volčic [85] proves that three non-collinear point sources are sufficient for determination. Except for Falconer's, these results only demonstrate the uniqueness of $K$ and are thus non-constructive. Other results include [46, 47, 57].

These theorems have been derived for convex sets, not the more restricted set of convex polygons. In this paper, we have only been concerned with polygons. It is not yet clear whether some results for convex sets may be obtained from those on convex polygons by suitable approximations.

First, we show that two parallel x-rays are not sufficient to determine a convex polygon, where we now use 'determine' in the usual interactive sense. We will use an adversary argument to show that there will be at least two convex polygons $P$ which satisfy the results of two parallel probes, regardless of how they are selected. Let the first probe return the image of a trapezoid $\tau_{1}$ and the second probe, aimed at an angle $\theta$ with respect to $l$, return a similar trapezoid $\tau_{2}$. Figure 13 shows the construction of two quadrilaterals which both give rise to images $\tau_{1}$ and $\tau_{2}$. Thus two parallel probes do not suffice to determine $P$.

Three parallel probes are sufficient to determine a convex polygon. Consider two orthogonal probes. From the first, we will obtain the complete set of $\mathrm{x}$-coordinates of vertices in $P$. From these we can determine $x_{\min }$, the smallest distance between distinct $\mathrm{x}$-coordinates. Note that up to two vertices may lie on any line of the form $x=c$. From the second probe we 
Figure 13: Two parallel probes are not sufficient for interactive determination.

will determine the complete set of y-coordinates of vertices and thus $y_{\max }$, an upper bound on the length of intersection between any line $y=c$ and $P$.

Aiming the third probe with angle $\alpha, \pi / 2-\arctan \left(x_{\min } / y_{\max }\right)<\alpha<\pi / 2$ will insure that no two vertices will be incident upon the same histogram line. This is steep enough so that no line with angle $\alpha$ will contain two of the old intersections. A linear sweep through the histogram vertices from the first and third probe will permit the intersections to be computed in $O(n)$ time.

For the probing models discussed earlier, the ability to interactively select probing directions is what made finite probing strategies possible. Aggregate probes are powerful enough to wave this restriction. However, let $P$ be a $2 n$-gon with equal sized isoscelese-triangular "bites" taken out of every other corner. Then two distinct orientations of $P$ exist where each direction is perpendicular to the base of a cut, and as Figure 14 shows for both of these every probe image is identical.

Origin probes present a different set of problems, which to solve we will need some earlier results. Recall Section 4.2 where we showed that the complete set of x-ray probes through $O$ determines a "spider-web" $S_{O}(P)$, which can be inverted such that any non-parallel edge pair of $P$ is determined up to rotation by $\pi$. It is this ability to determine edge pairs which makes origin probes more powerful than parallel probes. Two origin probes are necessary and sufficient to determine a convex polygon.

To show that two origin probes are sufficient, let $O_{1}$ be the origin of our first origin probe. From the previous discussion, it is clear that if the resulting spider web indicates no parallel 
Figure 14: Directions from regular polygons do not suffice for determination.

Figure 15: The two cases with all parallel edge pairs.

edges (ie. a degenerate linear segment of $S_{O_{1}}(P)$ ), that $P$ is determined up to rotation and that a second origin probe can easily be selected to distinguish between the possibilities $P$ and $\bar{P}$.

Further, $P$ is determined up to central reflection, or equivalently rotation through $\pi$, if there is even one non-parallel edge pair in $S_{O_{1}}(P)$ since this pair of edges is determined. The neighboring parallel edge pair has their slope $m$ and the distance between them determined by the inversion formula. A vertex of this edge is determined from the known edge pair and with $m$ gives the next edge. Walking around in this way determines $P$.

The only cases remaining are illustrated in Figure 15. Either $O$ is within $P$ or it is outside $P$. If $O$ is within $P$, all edge pairs are parallel which implies that $P$ is centrally symmetric with center $O$. However, for such a polygon $P=\bar{P}$ and the polygon is determined. In the second case, $O$ is outside $P$ and $P$ may be located anywhere in the angle sectors defined by $P$. By convexity, there is at most one parallel edge pair of $P$, and the other edges are defined by directions through $O$. With the slopes of all four edges known, a second point can be selected to yield a non-parallel edge-pair and determine $P$. 
Figure 16: Invisible vertices in star-shaped polygons.

Aggregate probes make possible the determination of a larger class of polygons than for single probes, since aspects of the entire polygon are recorded in each probe. However, one important property of parallel probes for convex polygons does not hold for star-shaped polygons, namely that each vertex of $P$ lies on a line determined by each probe. Figure 16 shows how vertices in star-shaped polygons can be invisible to parallel probes. Gardner [23] shows that star-shaped polygons cannot be determined (in the terminology of Hammer's X-ray problem) with constant number of probes, but the implications of this result on the interactive reconstruction of star-shaped polygons is unclear.

This problem of invisible vertices leads to the study of $k$-projections, discussed in [77]. A $k$-projection of a set of $n$ points is an orthogonal projection that yields at most $k$ point images. The hope is that further study of $k$-projections will provide insight into how many invisible vertices can possibly remain after $m$ probes.

\section{$5 \quad$ Half-Space Probes}

One of the most famous stories in the history of science is that of Archimedes figuring out how to test if the King's crown was made of solid gold as ordered or if the goldsmith cheated and added silver to the metal. The problem revolved around how to measure the volume of the irregularly shaped crown. Archimedes had the inspiration, while taking a bath, of measuring how much water the crown would displace and comparing it to the volume of water displaced an equal weight of gold. On making this discovery, he was so excited he ran naked through the streets of Syracuse yelling "Eureka". This tale provides inspiration for the study of half-space probes.

We define a half-plane probe to be the area of intersection between a closed half-plane $h$ and a polygon $P$. Let $h(l)$ be defined as the area of intersection between $P$ and the closed half-plane to the left of the directed line $l$. This notion can be generalized to half-space probes in higher dimensions, where each probe returns the volume of $P$ which intersects with the half-space. There is a close relationship between x-ray and half-plane probes which can be exploited to develop a linear half-plane probing strategy.

As mentioned above, the original inspiration for studying half-plane probes was the famous story of Archimedes measuring the volume of water the crown displaced. Such dunks 
in the tub are really half-space probes. More importantly, half-plane probing problems have application to tomography and remote sensing, such as the lunar occultation observations used to map astrostellar radio sources [81]. The instruments for measuring such radio sources have a lower resolution than desired, so each measurement represents the total amount of energy over an area. By waiting until the moon passes over a portion of the region and measuring how much the energy is reduced, detailed maps of the source can be produced. This is very close to our notion of a half-plane probe.

Half-plane probes have the advantage over other types of probes we have considered that they in some sense reflect the entire structure of the polygon in every probe. Thus they provide the possibility of extending probing results to simple polygons, since unlike with finger and x-ray probes concave edges are potentially verifiable.

\subsection{Upper and Lower Bounds}

To obtain absolute information about $P$ from half-plane probes, it is necessary to think in terms of groups of probes which work together. These are the same classes as defined for $\mathrm{x}$-ray probes, and are again designed to reflect the complementary goals of recognizing and determining edge pairs.

The first class of probes are origin probes, a set of half-plane probes bounded by lines all aimed through a common point $O$ within the object. Any half-plane probe which intersects a convex polygon and avoids its vertices will go through exactly two edges of the object.

Each half-plane is defined by a directed line. We can therefore consider the complete set of origin probes through a point $O=(0,0)$ as defined by $l_{t}: y=t x$, where $t=\tan (\theta)$, $2 \pi \leq \theta \leq 0$. These define a function $f(t)=h\left(l_{t}\right)-h\left(l_{0}\right)$. This function will contain enough information to determine the edges probed through, except for special cases. Here, we consider $f(t)$ for a wedge defined by two lines and containing the origin.

Theorem 3 Let $l_{1}: y=m_{1} x+b_{1}$ and $l_{2}: y=m_{2} x+b_{2}$ be two distinct lines, $m_{1}, m_{2}, b_{1}, b_{2} \neq$ $0, P$ be the unbounded wedge between $l_{1}$ and $l_{2}$ containing the origin, and let $y=f(t)$ be defined as above. Then

$$
A y t^{2}+B y t+C y+D t^{2}+E t=0,
$$

where $A=2 m_{1} m_{2}, B=-2\left(m_{1}^{2} m_{2}+m_{1} m_{2}^{2}\right), C=2 m_{1}^{2} m_{2}^{2}, D=b_{2}^{2} m_{1}-b_{1}^{2} m_{2}$, and $E=m_{2}^{2} b_{1}^{2}-m_{1}^{2} b_{2}^{2}$.

Proof: Consider the situation in Figure 17, where both edges intersect the $x$-axis. This involves no loss of generality, since a rotation of the axes can always be performed. Hence, we need not consider the case where either slope is 0 . For any $t$, the area swept out between $y=0$ and $y=t x$ is the sum of the areas of the two triangles defined by $y=0, y=t x$, and either $l_{1}$ or $l_{2}$. The value of $y=f(t)$ is defined to be the difference in area between the two triangles, $y=A_{1}-A_{2}$. More formally,

$$
y=\frac{t b_{1}{ }^{2}}{2 m_{1}\left(m_{1}-t\right)}-\frac{t b_{2}{ }^{2}}{2 m_{2}\left(m_{2}-t\right)} .
$$


Figure 17: Defining $f(t)$, the probes through an edge pair.

Multiplying through by the denominators and simplifying gives the result.

We note that $f(t)$ is infinite and hence ill-defined when $t$ is between $m_{1}$ and $m_{2}$. For example, when $t=m_{1}$ or $t=m_{2}, f(t)$ reduces to $m_{1}=m_{2}$. This complication does not occur when probing polygons since additional edges occur in this range. We will use Lemma 3 to determine the equations of the lines that contain edge pairs. If we have some number of origin probes through a common edge pair, then we can determine the $f(t)$ through the associated points. From $f(t)$, we then deduce the equations of the lines.

The function $f(t)$ is determined by five constants: $A, B, C, D$, and $E$. It follows that, in general, five probes through a pair of edges are enough to determine the function. Since all five constants are functions of the four line parameters they cannot all be independent. Indeed, $C=A^{2} / 2$. Given $A, B, D, E$, we can solve for the parameters of the equations:

$$
\begin{gathered}
m_{1}=m_{2}=\frac{-B \pm \sqrt{B^{2}-2 A^{3}}}{2 A} \\
b_{1}^{2}=\frac{E+m_{1} D}{m_{2}\left(m_{2}-m_{1}\right)}, \quad b_{2}{ }^{2}=\frac{E+m_{2} D}{m_{1}\left(m_{2}-m_{1}\right)} .
\end{gathered}
$$

From these equations several limitations on our ability to reconstruct the edges become apparent. Since $b_{1}$ and $b_{2}$ are squared, we obtain no information on the sign of the intercepts. Neither $m_{1}$ or $m_{2}$ are distinguished from each other, meaning we cannot associate which intercept belongs to which line. More serious is that $b_{1}$ and $b_{2}$ are undefined when $m_{1}=m_{2}$. Thus any probing strategy using origin probes must take special action on parallel edges.

However, to exploit Lemma 3 we must insure our probes intersect the same edge pair. Unfortunately, extra probes lying on $f(t)$ are not sufficient to verify $f(t)$. Parallel probes are a set of half-plane probes defined by lines of identical slope and direction. A complete collection of parallel probes of a given slope $\theta$ results in a cumulative area histogram $C(P, \theta)$ 
Figure 18: A polygon with $C(P, \theta)$ and its derivative.

Figure 19: Determining vertices of $P$.

of the area of the object. The derivative of $C$ at any point gives the value of the x-ray probe defined by the probing line. The complete derivative of $C$ gives the result of a parallel x-ray aggregate probe as shown in Figure 18. By analogy with x-ray probes, they provide a mechanism for verifying edge pairs. Four parallel half-plane probes through an edge pair are sufficient to verify the edge pair, where three x-ray probes sufficed for the same task.

As shown in [78], $3 n+15$ probes are sufficient to determine the first edge pair and a point on the boundary of $P$, with up to $3 n-3$ parallel probes defining a section to origin probe, and the added complication of bounding the height of the polygon within this section, so the probes intersect $P$ within this section.

Probes through known points on the boundary of $P$ are defined as boundary probes. We can use boundary probes to develop a more efficient probing strategy through the observation that three parallel probes through an edge pair are sufficient to determine the second edge, if one edge is known to be contained in $l_{1}: m x+b$.

After determining an edge pair, we have the situation in Figure 19. The edges contain known points $p_{1}, p_{2}$ and $q_{1}, q_{2}$. We conjecture the edges meet at $v$. To test this, we need a probe through $q_{1}$ and $p_{2}$. If it returns the area of triangle $\left(q_{1}, p_{2}, v\right)$, we have verified vertex $v$, otherwise, there is at least one additional edge in the unexplored corner. Let $v$ be the point on $l_{1}$ such that the result of this probe equals $\alpha\left(q_{1}, p_{2}, v\right)-\alpha\left(q_{2}, \hat{v}, v\right)$, where we define $\alpha(a, b, c)$ to be the area of the triangle defined by the three points. 
Thus edge $\left(p_{1} p_{2}\right)$ cannot extend past $v$ without violating convexity. Probing parallel to $q_{1} v$ between $q_{1}$ and $q_{2}$ we intersect a new edge pair, one of which is $\left(q_{1} q_{2}\right)$.

We can parallel probe this section, and then consider these probes as boundary probes once we have determined an edge pair with $\left(q_{1} q_{2}\right)$ as the known line. Aim the $i$ th parallel probe between the $(i-1)$ st and the $(i-2)$ nd parallel probe. If it takes more than five parallel probes to verify an edge pair, we have identified another section to parallel probe.

We shall pivot around edge $\left(q_{1} q_{2}\right)$, repeatedly determining the edge in the rightmost unexplored section. Since the initial probe to verify the vertex corresponds to one of the origin or parallel probes used previously, we only need four additional probes each to determine the rest of the edges.

This completes the determination strategy for convex $n$-gons. As discussed above, $3 n+15$ probes are sufficient to determine the first two edges. From the preceding discussion, 4 probes are sufficient to determine each additional edge. Thus the total number of probes required is $(3 n+15)+4(n-2)=7 n+7$.

A lower bound of $2 n$ half-plane probes can be based on topological arguments [78]. We can represent a convex polygon $P$ as a point in $2 n$-dimensional space. Every point in an $\epsilon$ neighborhood around $P$ represents a slightly different convex polygon.

Lefschetz [48] proves that a region of a Euclidean $m$-space cannot be parameterized by less than $m$ parameters. Since the result of a probe is an algebraic function of $P$ it can be considered to be a parameter of $P$. Thus at least $2 n$ probes are necessary to determine a convex polygon.

The following argument shows that $n+1$ half-plane probes are sufficient to verify a convex $n$-gon. For one of the edges of $P$, probe in both directions of the line containing the edge. For the remaining $n-1$ edges, probe once along the defining line. With each edge, we know $P$ entirely lies within each of $n$ half-planes. The intersection of these half-planes is $P$. Since the intersection of these half-planes has exactly the area of $P$, we have verified $P$. Note that fewer half-plane probes are sufficient for verification than for finger or x-ray probes. In [78], it is shown that at least $2 n / 3$ half-plane probes are necessary to verify a convex $n$-gon.

Two more interesting generalizations of half-plane probes to higher dimensions prove to be much more difficult to analyze. Consider a cross-sectional area probe which, for a given plane in $E^{3}$ returns the area of intersection with $P$. This differs from the half-plane probe described above in that the line which defines the appropriate half-plane is at $\infty$. The results in this paper do not appear to help with cross-sectional area probes, since they rely on isolating a section of $P$ containing only two facets, which is not possible with a full cross-section of $P$. Even the problem of determining a tetrahedron in a constant number of probes is open and appears difficult.

The other interesting generalization would be to half-space probes in $E^{3}$, which for a specified half-space returns the volume of intersection. The simpler problem of determining tetrahedra is also open for half-space probes. 


\section{Oracles and Learning}

The problems we have discussed thus far in this paper have all involved probing purely geometric objects, usually convex polytopes. But the notion of probing leads to interesting problems even if we stretch the definition of geometric object.

In the theory of computation [41], an oracle is a black box which can answer certain questions in one unit of time. Augmenting a model of computation with a particular oracle produces a potentially more powerful one, and so studying these models provides a framework for studying the power of the oracle. The probing models we have discussed can be thought of as oracles, and we have shown that different models require different number of probes for determination. Considering more general oracles extends the notion of interactive reconstruction to different types of problems.

\subsection{Optimization Problems and Algorithmic Paradigms}

Hyperplane probes have a close connection to optimization problems, since the constraints for mathematical programs are typically represented by hyperplanes. We shall look more closely at this connection, as well as at hyperplane probes as a paradigm for solving algorithmic problems.

The results of a hyperplane probe can be simulated by a linear program, assuming each facet of the polytope is described by the equation of the hyperplane supporting its faces. Let $d$ be the number of dimensions of $P$. Each facet $i$ is represented by one constraint:

$$
c_{1 i} x_{1}+c_{2 i} x_{2}+\ldots+c_{d i} x_{d} \leq c_{(d+1) i}
$$

The objective function $F(x)$ describes the moving hyperplane, which is specified by the vector $\left(a_{1}, a_{2}, \ldots, a_{d}\right)$, is

$$
F(x): a_{1} x_{1}+a_{2} x_{2}+\ldots+a_{d} .
$$

The result of the probe is determined by maximizing or minimizing $F(x)$, depending upon whether the probe originates from $+\infty$ or $-\infty$. This value is the intercept which with $F(x)$ completely specifies the hyperplane.

These simulated probes are actually more powerful than the previously defined hyperplane probes, since the contact vertex is specified by the optimal vector $x$.

A problem of interest in combinatorial optimization can be solved using these simulated probes. Given a convex polytope $P$ containing the origin $O$, what vertex of $P$, $v_{\max }$, is furthest from $O$ ? This can be easily formulated as a quadratic program, with the previous set of constraints and maximizing the objective function

$$
x_{1}^{2}+x_{2}^{2}+\ldots+x_{d}^{2}
$$

Unfortunately, the problem of solving quadratic programs is NP-hard [25] and Rajan and Taylor [82] have considered using simulated probes to determine $v_{\max }$. If all vertices of $P$ are equidistant from $O, P$ will need be determined. Since the number of vertices can be 
exponential in the number of constraints this is not efficient, but it is reasonable to consider heuristics to select the probes.

Determining an approximation to the volume of an object has been considered for a slightly different computational model. Bárány and Füredi [5] consider an oracle which for a point $x$ tests whether $x \in P$, where $P$ is a convex body and seek to determine upper and lower bounds to the volume of $P$. They prove that no polynomial time algorithm exists such that the ratio of these quantities will be less than $(d / \log d)^{d}$ in $E^{d}$. Elekes [18] proves the combinatorial result that the volume of the convex hull of $n$ points on a unit sphere in $E^{d}$ is at most $n / 2^{d}$, which leads directly to results for finger and hyperplane probes. Gritzman, Klee, and Westwater [35] consider several problems on using probes to construct bounded polytopes which contain $P$, which imply an upper bound on the volume.

If the coefficients and variables in a linear program are restricted to integer values, the result is an integer program. Although the problem of solving an integer program is NPcomplete, its great practical importance has lead to the development of a variety of techniques for solving integer programs. One class of techniques, the cutting-plane algorithms [63], relaxes the problem by considering the equivalent linear program and repeatedly adds constraints or cutting planes which prunes the feasible region without removing any integer lattice points. The algorithm terminates when the optimal point of the revised linear program has integer coordinates. These cutting-planes are in fact hyperplane probes, and an integer programming algorithm results from using simulated hyperplane probes to determine the convex hull of lattice points beneath the feasible region.

\subsection{Cut-set Probes}

Consider a graph $G=(V, E)$ whose $n$ vertices are points in general position in the plane (that is, no three points are collinear) and whose edges are all straight line segments between pairs of vertices. We assume that the positions of the vertices are known, but nothing about the edges is specified. A cut-set probe returns the number of edges cut by a specified line.

It is not obvious that cut-set sizes should permit reconstruction. Complete knowledge of other graph parameters, such as degree sequences do not have this property. A problem similar to ours involves the complexity of determining properties of graphs given queries of the form "is edge $\{i, j\}$ in the graph?". Karp has conjectured that monotone graph properties such as connectivity are evasive, meaning that in the worst case all edges must be queried to determine whether the graph has this property. Lower bound results [44, 68] show such properties are within a multiplicative constant of being evasive.

Reconstructing graphs from cut-set probes is evasive in a similar sense, since it can be shown [76] that $\left(\begin{array}{l}n \\ 2\end{array}\right)$ cut-set probes are necessary and sufficient for edge reconstruction. In particular, a specific convex imbedding of the graph $K_{n / 2, n / 2}$ requires all cutsets for reconstruction. If the size of an arbitrary cutset is used as the probe, $\theta\left(n^{2} / \log n\right)$ probes are necessary and sufficient, with the lower bound following from information theory and the upper bound a consequence of results for a more general oracle [1].

An interesting, although unrelated, graph probing problem comes directly from the notion of electrical tomography [28]. In electrical tomography, a number of pairs of probe points 
are made and the electrical resistance between the two points is measured. The measured resistance of each will be a function of the entire organ, not just the line between the two points. This can be modeled by assuming a graph between the points, such that each arc of the graph contains a fixed resistor. Further, the network obeys the classical laws for parallel and series resistance. The problem, given such a network, is how many probes are necessary to determine the resistors associated with each edge.

It is not obvious that network can be determined for all graphs. Gilbert and Shepp [28] claim that the resistances can be determined if the graph is complete. As a counter-example to the general result, they present three networks of five vertices and eight edges which yield identical measurements.

\subsection{Learning}

Building systems which learn is a fundamental goal of artificial intelligence, but properly evaluating a learning system requires a rigorous definition of the terms "concept" and "learning". Often, concepts are modeled as regions in a $d$-dimensional space. For the concept of "average build", people with height from 5'5" to 6'0" and weight from 135-200 lbs., could be defined as a rectangular region in a height-weight space. Learning can be viewed as inducing such a concept from a set of examples.

The key question in the theory of learning is how difficult it is to learn a particular class of concepts. Beginning with Valiant's theory of learning [83], there has been considerable effort in defining models of learning of different types of concepts (Boolean formulae, finite automata, geometric regions, etc.) under a variety of conditions (random examples, positive examples, negative examples, etc.). Under various models, it can be shown that certain classes of concepts can be learned more efficiently than others. It is beyond the scope of this paper to discuss these models in any detail, but see $[6,9]$ as representative papers in the theory of learning.

When the student is allowed to ask questions, learning a concept becomes a probing problem. Maass and Turán [56] consider such a model, where the student proposes a region consistent with previous queries, and the teacher provides a counterexample - a point which either should or should not be in the region. By being clever, the student can minimize the number of questions needed to determine the region. As an example [56], consider the class of concepts defined by boxes in $d$ dimensions, where the boundary of a box is specified by points with integer coordinates between 0 and $n$. A naive algorithm might require $\mathrm{O}\left(n^{d}\right)$ queries, but if the student uses a variant of binary search, they can propose regions consistant with all previous queries which will eliminate a fraction of the points outside the concept region reguardless of the counterexample selected by the teacher. Since a fraction of the outlying points are identified in each iteration, $\mathrm{O}(d \log n)$ probes are sufficient to determine the box.

Romanik and Smith [69] introduce the notion of the testability of geometric object classes, based on Valiant's learning model. Their testability results for convex polygons are based on Cole and Yap's $2 n$ verification strategy for convex polygons. 


\section{Conclusions}

Our paper [75] provides a list of seventeen open problems in probing. Since its appearance there has been substantial progress on five of the problems. Lindenbaum and Bruckstein [53] have obtained tight bounds on the problem of minimizing the number of rounds required for determination, when $k$ simultaneous finger probes may be made per round (Problem 13). A $3 n-3$ lower bound for determination with x-ray probes follows from some related results [54] (Problem 6), which gives the surprising result that access to both finger and x-ray probes provides no significant increase in power over access to finger probes alone (Problem 15). Joseph and Skiena [43] give optimal strategies for model-based probes (Problem 4) and Gardner [23] considers x-ray probes and star-shaped polygons (Problem 8).

Of the remaining problems, the following are most important and should be the focus of further research in geometric probing.

1. Can the techniques of x-ray probes be applied to real tomographic systems? We believe they can. The goal in geometric probing is to minimize the number of measurements required for reconstruction. In the context of tomography, this translates to minimizing the amount of radiation the patient is exposed to.

The most important reconstruction algorithms are transform methods, which are direct implementations of the Radon inversion formula. Since the theory is based on having the complete set of line integrals, interpolation techniques are used to approximate this from the set of observations. There is a close relationship between Radon and Fourier transforms which is exploited in these algorithms.

We believe that algebraic reconstruction techniques [38] have more potential when the probing directions can be interactively selected. Since the final image to be displayed will be a matrix of pixel values, algebraic techniques view reconstruction as an optimization problem, with each pixel as a different variable. Each probe represents a linear constraint, where the weighted sum of the pixels intersected by the probe is set equal to the value of the line integral. Due to insufficient data or errors leading to inconsistent constraints, this is typically solved as an optimization problem, according to some criteria such as least squares. Additional constraints to produce certain smoothness properties can also be included.

2. Tightening the gap between our lower and upper bounds for determination for x-ray probes. The lower bound for determination has been raised from $3 n / 2$ [17] to $2 n$ [78] to, most recently, $3 n-3$. The best upper bound remains $5 n+19$, but we conjecture that $3 n+c$ probes are necessary and sufficient, since up to $2 n$ probes in our strategy are wasted locating the first edge pair.

Tightening these bounds is important, because they suggest that all probing models may require $\sim 3 n$ probes for reconstruction, and thus are equivalent in power.

3. How many x-ray parallel x-rays are necessary to determine a star-shaped $n$-gon? This generalizes Hammer's problems beyond convex polygons. It has recently been shown 
by Gardner [23] that there does not exist a constant $k$ such that $k$ predetermined probes are sufficient to determine an arbitrary star-shaped polygon, but this does not preclude the existence of an algorithm for interactive reconstruction.

4. Tighten Dobkin, Edelsbrunner, and Yap's [16] gap between the lower and upper bounds for determination in higher dimensions. Associated with this problem is the time complexity of finger probing in higher dimensions.

5. Silhouette probes return the shadow cast by a polytope in a specified direction. These dualize to cross-section probes which return a slice of the polytope. Tighten the current bounds [16] on determination with silhouettes in $E^{3}$.

6. Consider generalizations of half-plane probes to higher dimensions. A half-space probe returns the volume of intersection between a polytope $P$ and the half-space. A crosssectional area probe returns the area of intersection between a plane and $P$. Is there a finite strategy for reconstructing convex polyhedra in $E^{3}$ from half-space or crosssectional area probes?

Since each half-plane probe can be considered as a function of the entirety of $P$, it is reasonable to consider generalizing to a wider class of objects. Does there exist a finite probing strategy for reconstructing star-shaped polygons from half-plane probes? Such a strategy cannot exist for x-ray probes.

7. How many cut-set probes are needed to determine the number of edges or degree sequence in a graph? Are fewer probes necessary for special types of graphs, such as planar graph embeddings? $O(n \log n)$ arbitrary cut-set probes are sufficient to determine a tree, while lower bounds can be based on enumeration results [37] for the class of graph.

\section{Acknowledgements}

I would like to thank Herbert Edelsbrunner for calling my attention to the problem of probing and his patient advice and teaching. I would also like to thank Eugene Joseph and the referees for reading earlier versions of this manuscript. R. J. Gardner provided important feedback on Section 4.3.

\section{References}

[1] A. Aggarwal, D. Coppersmith, and D. Kleitman. A generalized model for understanding evasiveness. Info. Proc. Letters, 30:205-208, 1989.

[2] P. D. Alevizos, J. D. Boissonnat, and M. Yvinec. An optimal $\mathrm{O}(n \log n)$ algorithm for contour reconstruction from rays. Proc. Third ACM Symposium Computational Geometry, pages 162-170, 1987. 
[3] P. D. Alevizos, J. D. Boissonnat, and M. Yvinec. On the order induced by a set of rays: Application to the probing of non-convex polygons. Proc. IEEE Conf. on Robotics and Automation, pages 202-207, 1989.

[4] P. D. Alevizos, J. D. Boissonnat, and M. Yvinec. Non-convex contour reconstruction. J. Symbolic Computation, to appear.

[5] Imre Bárány and Z. Füredi. Computing the volume is difficult. Discrete and Computational Geometry, 2:319-326, 1987.

[6] E. Baum. On learning a union of half-spaces. J. Complexity, 6:67-101, 1990.

[7] P. Belleville. Probing polygons minimally is hard. In Proc. Third Canadian Conference on Computational Geometry, 1990.

[8] Herbert J. Bernstein. Determining the shape of a convex $n$-sided polygon by using $2 n+k$ tactile probes. Information Processing Letters, 22:255-260, 1986.

[9] A. Blumer, A. Ehrenfeucht, D. Haussler, and M. Warmuth. Learnability and the VapnikChervonenkis dimension. J. ACM, 36:929-965, 1989.

[10] J. D. Boissonnat. Shape reconstruction from planar cross-sections. Computer Vision, Graphics, and Image Processing, 44:1-29, 1988.

[11] J. D. Boissonnat and M. Yvinec. Probing a scene of non-convex polyhedra. Proc. Fifth ACM Symposium on Computational Geometry, pages 237-246, 1989.

[12] J. D. Boissonnat and M. Yvinec. Probing a scene of non-convex polyhedra. Technical report, INRIA, October 4,1989.

[13] B. Chazelle, H. Edelsbrunner, and L. J. Guibas. The complexity of cutting convex polytopes. Proc. 19th ACM Symposium on the Theory of Computing, pages 66-76, 1987.

[14] B. Chazelle, L. J. Guibas, and D. T. Lee. The power of geometric duality. BIT, 25:76-90, 1985.

[15] Richard Cole and Chee K. Yap. Shape from probing. Journal of Algorithms, 8:19-38, 1987.

[16] David P. Dobkin, Herbert Edelsbrunner, and Chee K. Yap. Probing convex polytopes. Proc. 18th ACM Symposium on the Theory of Computing, pages 424-432, 1986.

[17] H. Edelsbrunner and S. S. Skiena. Probing convex polygons with x-rays. SIAM J. Computing, 17:870-882, 1988.

[18] G. Elekes. A geometric inequality and the complexity of computing volume. Discrete and Computational Geometry, 1:289-292, 1986. 
[19] R. E. Ellis, E. M. Riseman, and A. R. Hanson. Tactile recognition by probing: Identifying a polygon on a plane. Proc. Amer. Assoc. of Art. Intelligence Conf., pages 632-637, 1986.

[20] K. J. Falconer. Hammer's x-ray problem and the stable manifold theorem. J. London Math. Soc. (2), 28:149-160, 1983.

[21] K. J. Falconer. X-ray problems for point sources. Proc. London Math Soc. (3), 46:241262, 1983.

[22] R. J. Gardner. Symmetrals and x-rays of planar convex bodies. Arch. Math, 41:183-189, 1983.

[23] R. J. Gardner. X-rays of polygons. Technical report, Istituto Analisi Globale e Applicazioni, 1990.

[24] R. J. Gardner and P. McMullen. On Hammer's x-ray problem. J. London Math. Soc., 21:171-175, 1980.

[25] M. R. Garey and D. S. Johnson. Computers and Intractability: A Guide to the Theory of NP-Completeness. W. H. Freeman, San Francisco CA, 1979.

[26] Peter C. Gaston and Tomas Lozano-Perez. Tactile recognition and localization using object models: The case of polyhedra on a plane. IEEE Transactions on Pattern Analysis and Machine Intelligence, PAMI-6:257-266, May 1984.

[27] O. Giering. Bestimmung von eibereichen und eikörpern durch Steiner symmetrisierungen. Sber. Bayer. Akad. Wiss. München, Math.-Nat. Kl., pages 225-253, 1962.

[28] E. N. Gilbert and L. A. Shepp. Electrical tomography. In T. M. Cover, editor, Open Problems in Communication and Computation, pages 164-170. Springer-Verlag, New York, 1987.

[29] J. F. Greenleaf. Computerized tomography with ultrasound. Proc. IEEE, 71:330-337, 1983.

[30] John Paul Greschak. Reconstructing Convex Sets. PhD thesis, MIT Department of Electrical Engineering and Computer Science, Cambridge MA, 1985.

[31] W. Eric L. Grimson. The combinatorics of local constraints in model-based recognition and localization from sparse data. J. ACM, 33:658-686, 1986.

[32] W. Eric L. Grimson. Sensing strategies for disambiguating among multiple objects in known poses. IEEE Journal of Robotics and Automation, RA-2:196-213, December 1986. 
[33] W. Eric L. Grimson and Tomas Lozano-Perez. Model-based recognition and localization from sparse range or tactile data. International Journal of Robotics Research, 3:3-35, Fall 1984.

[34] W. Eric L. Grimson and Tomas Lozano-Perez. Localizing overlapping parts by searching the interpretation tree. IEEE Transactions on Pattern Recognition and Machine Intelligence, PAMI-9:469-482, July 1987.

[35] P. Gritzmann, V. Klee, and J. Westwater. On the limited power of linear probes and other optimization oracles. Proc. Sixth ACM Symposium Computational Geometry, pages 92-101, 1990.

[36] P. C. Hammer. Problem 2. In Proc. Symposium Pure Math vol VII: Convexity, pages 498-499. Amer. Math. Soc., 1963.

[37] Frank Harary and Edgar M. Palmer. Graphical Enumeration. Academic Press, New York, 1973.

[38] Gabor T. Herman. Image Reconstruction from Projections: the Fundamentals of Computerized Tomography. Academic Press, New York, 1980.

[39] Gabor T. Herman. The special issue on computerized tomography. Proc. IEEE, 71:291292, March 1983.

[40] W. S. Hinshaw and A. H. Lent. An introduction to NMR imaging: from the Bloch equation to the imaging equation. Proc. IEEE, 71:338-350, 1983.

[41] J. Hopcroft and J. Ullman. Introduction to Automata Theory, Languages, and Computation. Addison-Wesley, Reading MA, 1979.

[42] Berthold Klaus Paul Horn. Robot Vision. MIT Press, Cambridge MA, 1986.

[43] E. Joseph and S. Skiena. Model-based probing strategies for convex polygons. Technical Report 90-35, SUNY Stony Brook Department of Computer Science, Stony Brook NY, 1990.

[44] Valerie King. Lower bounds on the complexity of graph properties. Proc. 20th ACM Symposium on the Theory of Computing, pages 468-476, 1988.

[45] G. F. Knoll. Single-photon emission computed tomography. Proc. IEEE, 71:320-329, 1983.

[46] D. Kölzow, A. Kuba, and A. Volčič. An algorithm for reconstructing convex bodies from their projections. Discrete and Computational Geometry, 1987.

[47] A. Kuba and A. Volčič. Characterization of measurable plane sets which are reconstructible from their two projections. Inverse Problems, 4:513-527, 1988. 
[48] Solomon Lefschetz. Introduction to Topology. Princeton University Press, Princeton NJ, 1949.

[49] S. Y. Robert Li. Reconstruction of polygons from projections. Information Processing Letters, 28:235-240, 1988.

[50] M. Lindenbaum. Topics in Geometric Probing. PhD thesis, Dept. of Electrical Engineering, Technion, Haifa, Israel, 1990.

[51] M. Lindenbaum and A. Bruckstein. Reconstructing convex sets from support hyperplane measurements. Technical Report 673, Dept. of Electrical Engineering, Technion, Haifa, Israel, 1988.

[52] M. Lindenbaum and A. Bruckstein. Blind approximation of planar convex sets. Technical Report CIS Report 9008, Dept. of Electrical Engineering, Technion, Haifa, Israel, 1990.

[53] M. Lindenbaum and A. Bruckstein. Parallel strategies for geometric probing. In IEEE Conf. on Robotics and Automation, Sacramento CA, April 1991.

[54] M. Lindenbaum and A. Bruckstein. Reconstruction of polygonal sets by constrained and unconstrained double probing. Annals of Mathematics and Artificial Intelligence, to appear.

[55] K. Lyons and D. Rappaport. An efficient algorithm for identifying objects using robot probes. Technical report, Department of Computing and Information Science, Queens University, Kingston, Ontario, Canada, January 1989.

[56] W. Maass and G. Turán. On the complexity of learning from counterexamples. In Proc. 30th IEEE Foundations of Computer Science, pages 262-267, 1989.

[57] G. Mägerl and A. Volčič. On the well-posedness of the Hammer x-ray problem. Annali di Matematica pura ed applicata (IV), CXLIV:173-182, 1986.

[58] Worthy Martin and J. K. Aggarwal. Volumetric description of objects from multiple views. IEEE Trans. Pattern Recognition and Machine Intelligence, PAMI-5:150-158, 1983.

[59] L. S. Narasimhan. Recognition of Polyhedral Objects: Concepts and Algorithms. PhD thesis, School of Management Science, University of Texas, Dallas TX, 1988.

[60] B. K. Natarajan. An algorithmic approach to the automated design of parts orienters. Proc. 27th IEEE Foundations of Computer Science Conf., pages 132-142, 1986.

[61] B. K. Natarajan. On detecting the orientation of polygons and polyhedra. Proc. Third ACM Symposium Computational Geometry, 3:146-152, 1987. 
[62] T. Okada and S. Tsuchiya. Object recognition by grasping. Pattern Recognition, 9:111119, 1977.

[63] Christos Papadimitriou and Kenneth Steiglitz. Combinatorial Optimization. PrenticeHall, Englewood Cliffs NJ, 1982.

[64] Nicholas Pippenger. Sorting and selecting in rounds. SIAM J. Computing, 16:1032-1038, 1987.

[65] J. Prince and A. Willsky. Reconstructing convex sets from support line measurements. IEEE Trans. Pattern Recognition and Machine Intelligence, PAMI-12:377-389, 1990.

[66] J. Radon. Über die bestimmung von funktionen durch ihre integralwerte langs gewisser manningfaltigkeiten. Ber. Verb. Sächs. Akad. Wiss., Leipzig, Math. Phys. Kl., 69:262$277,1917$.

[67] J. A. Reeds, L. A. Shepp, P. C. Fishburn, and J. C. Lagarias. Sets uniquely determined by projections. Technical report, AT\&T Bell Laboratories, Murray Hill, NJ, July 1986.

[68] R. Rivest and S. Vuillemin. On recognizing graph properties from adjacency matrices. Theor. Comp. Sci., 3:371-384, 1978.

[69] K. Romanik and C. Smith. Testing geometric objects. Technical Report CS-TR-2473, Dept. of Computer Science, University of Maryland, College Park, MD, May 1990.

[70] G. Rote. The convergence rate of the sandwich algorithm for approximating convex functions. Technical Report 118, Institute Für Mathematik, Technische Universität Graz, Graz, Austria, May 1990.

[71] Luis A. Santalo. Integral Geometry and Geometric Probability. Addison-Wesley, Reading MA, 1976.

[72] Jacob T. Schwartz and Micha Sharir. Some remarks on robot vision. Technical report, New York University, Courant Institute of Mathematical Sciences, New York, April 1984.

[73] L. A. Shepp and J. B. Kruskal. Computerized tomography: the new medical x-ray technology. Amer. Math. Monthly, 85:420-439, 1978.

[74] S. Skiena. Geometric Probing. PhD thesis, University of Illinois, Department of Computer Science, Urbana IL, 1988.

[75] S. Skiena. Problems in geometric probing. Algorithmica, 4:599-605, 1989.

[76] S. Skiena. Reconstructing graphs from cut-set sizes. Information Processing Letters, 32:123-127, 1989. 
[77] S. Skiena. Counting the number of $k$-projections of a point set. J. Combinatorial Theory, A, 55:153-160, 1990 .

[78] S. Skiena. Probing convex polygons with half-planes. J. Algorithms, 12:359-374, 1991.

[79] Kennan T. Smith, Donald C. Solomon, and Sheldon L. Wagner. Practical and mathematical aspects of the problem of reconstructing objects from radiographs. Bulletin Amer. Math. Soc., 83:1227-1270, November 1977.

[80] A. Spyridi and A. Requicha. Accessibility analysis for the automatic inspection of mechanical parts by coordinate measuring machines. Technical Report 257, Institute for Robotics and Intelligent Systems, University of Southern California, 1989.

[81] J. H. Taylor. Two-dimensional brightness distribution of radio sources from lunar occultation observations. Astrophys. J., 150:421-426, 1967.

[82] R. H. Taylor and V. T. Rajan. The efficient computation of uncertainty spaces for sensor-based robot planning. Technical Report 62814, IBM Watson Research Center, Yorktown Heights NY, September 8, 1988.

[83] L. Valiant. A theory of the learnable. Comm. ACM, 27:1134-1142, 1984.

[84] A. Volčič. A new proof of the Giering theorem. Suppl. Rendiconti Circolo Mat. Palermo, ser. II, 8:281-295, 1985.

[85] A. Volčič. A three-point solution to Hammer's x-ray problem. J. London Math. Soc. (2), 27:349-359, 1986.

[86] Y. K. Wang, M. J. Magee, and J. K. Aggarwal. Matching three-dimensional objects using silhouettes. IEEE Trans. Pattern Recognition and Machine Intelligence, PAMI6:513-518, 1984. 